\section{Wildlife as Source of Human Escherichia coli 0157 Infection}

\section{Brian Crook, Helena Senior}

Author affiliation: Health and Safety Laboratory, Health and Safety Executive, Buxton, United Kingdom

\section{DOI: https://doi.org/10.3201/eid2312.171210}

To the Editor: The article by Probert et al. (1) highlighted that wild animals (in this case, deer) can act as a reservoir of Shiga toxin-producing Escherichia coli (STEC) O157 infection. Our previous research (2) broadens this to include wild animals as STEC O157 carriers. In our study, an outbreak of STEC O157 infection in eastern England was epidemiologically linked to visiting a wildlife park. Unlike in petting zoos, the visitors had no direct contact with the animals. Transmission of infection was attributed to contact with the feces of wild rabbits (Oryctolagus cuniculus) in a play area; the rabbits had been in contact with STEC O157-positive cattle in an adjacent field (3). To prove this hypothesis, we identified rabbit populations living on farms with STEC O157-positive cattle. We trapped the rabbits humanely in cages, collected their feces during their confinement before release, and tested the feces for STEC O157 by culture and PCR. Of 97 samples collected in the summer, $8(8.2 \%)$ were positive by culture; these samples came from 4 of 6 farms in the study. By PCR analysis, 20 of $97(20.6 \%)$ samples were positive. None was positive during the winter, when cattle were housed indoors, suggesting a link between STEC O157 positivity in cattle and rabbits. In conclusion, when outbreaks of this serious human infection are linked to the rural environment, it is necessary to take wildlife into consideration, both as a reservoir of transmission and as carriers. Equally, persons should not underestimate the necessity of good hand hygiene if they have contact with wild animal feces.

\section{References}

1. Probert WS, Miller GM, Ledin KE. Contaminated stream water as source for Escherichia coli O157 illness in children. Emerg Infect Dis. 2017;23:1216-8. http://dx.doi.org/10.3201/eid2307.170226

2. Scaife HR, Cowan D, Finney J, Kinghorn-Perry SF, Crook B. Wild rabbits (Oryctolagus cuniculus) as potential carriers of verocytotoxin-producing Escherichia coli. Vet Rec. 2006;159: 175-8. http://dx.doi.org/10.1136/vr.159.6.175

3. Bailey JR, Warner L, Pritchard GC, Williamson S, Carson T, Willshaw $\mathrm{G}$, et al. Wild rabbits - a novel vector for Vero cytotoxigenic Escherichia coli (VTEC) O157. Commun Dis Public Health. 2002;5:74-5.

Address for correspondence: Brian Crook, Health and Safety Laboratory-Microbiology Team, Harpur Hill, Buxton SK17 9JN, UK; email: brian.crook@hsl.gsi.gov.uk

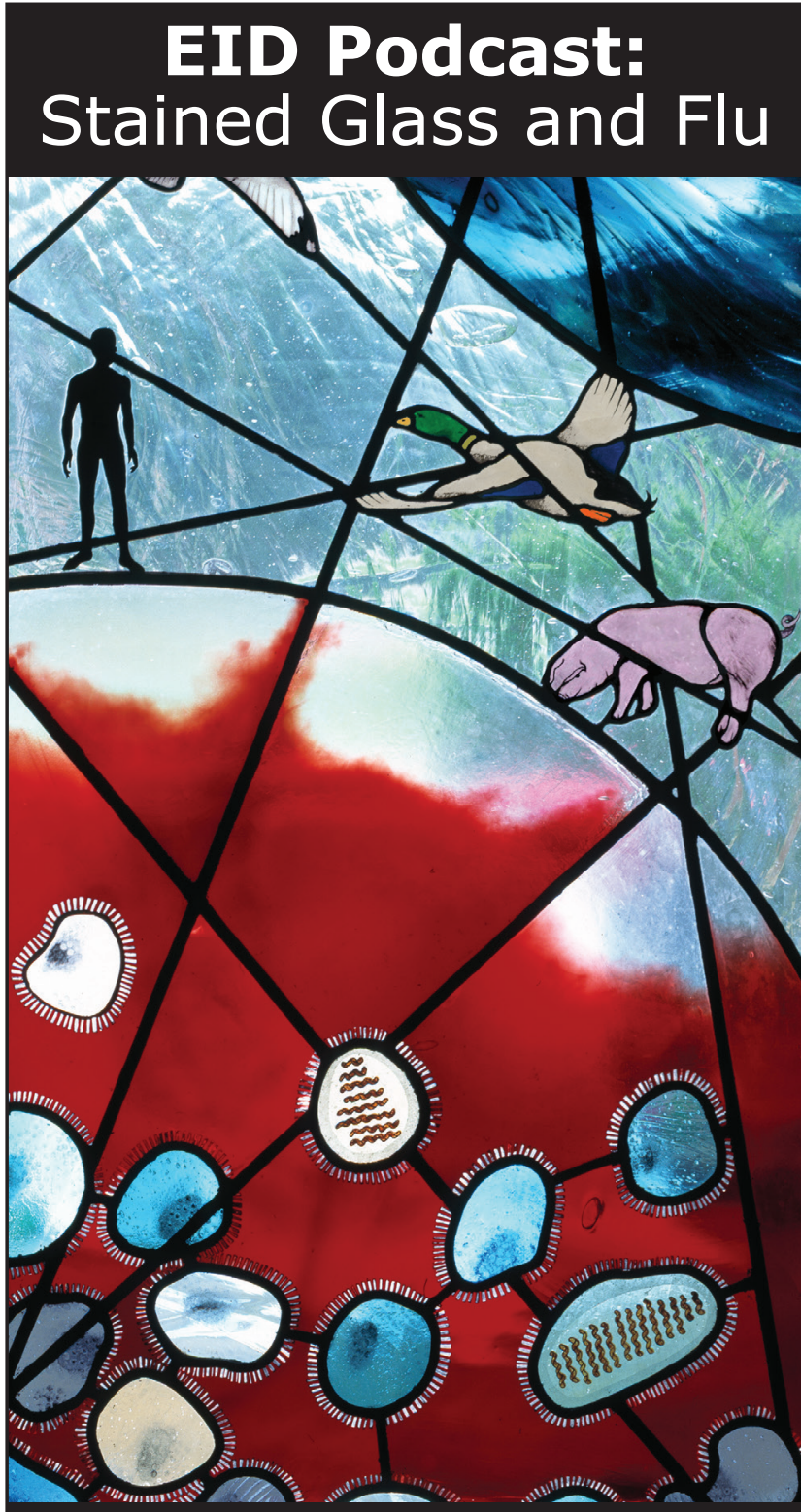

The work of art shown here depicts the interrelationship of human, animal, and environmental health.

Stained-glass windows have been appreciated for their utility and splendor for more than 1,000 years, and this engaging work of art by stained glass artist Jenny Hammond reminds us that influenza A viruses-which can be easily spread between animals and human, use various host species, and exist in many different environments-remain an enduring and global health concern.

\section{Visit our website to listen:} https://www2c.cdc.gov/ podcasts/player. asp?f $=\mathbf{8 6 4 4 9 5 0}$ 